\title{
Severe Hypomyelination of the Murine CNS in the Absence of Myelin-Associated Glycoprotein and Fyn Tyrosine Kinase
}

\author{
Karin Biffiger, ${ }^{1}$ Susanne Bartsch, ${ }^{1}$ Dirk Montag, ${ }^{1}$ Adriano Aguzzi, ${ }^{2}$ Melitta Schachner, ${ }^{3}$ and Udo Bartsch, ${ }^{1,3}$ \\ 1/nstitute for Neurobiology, Federal Institute of Technology, Hönggerberg, CH-8093 Zürich, Switzerland, 2/nstitute of \\ Neuropathology, University Hospital of Zürich, CH-8091 Zürich, Switzerland, and 3Zentrum für Molekulare Neurobiologie, \\ Universität Hamburg, D-20246 Hamburg, Germany
}

\begin{abstract}
The analysis of mice deficient in the myelin-associated glycoprotein (MAG) or Fyn, a nonreceptor-type tyrosine kinase proposed to act as a signaling molecule downstream of MAG, has revealed that both molecules are involved in the initiation of myelination. To obtain more insights into the role of the MAG-Fyn signaling pathway during initiation of myelination and formation of morphologically intact myelin sheaths, we have analyzed optic nerves of MAG-, Fyn- and MAG/Fyn-deficient mice. We observed a slight hypomyelination in optic nerves of MAG mutants that was significantly increased in Fyn mutants and massive in MAG/Fyn double mutants. The severe morphological phenotype of MAG/Fyn mutants, accompanied by behavioral deficits, substantiates the importance of both molecules for the initiation of myelination. The different severity of the phenotype of different
\end{abstract}

genotypes indicates that the MAG-Fyn signaling pathway is complex and suggests the presence of compensatory mechanisms in the single mutants. However, data are also compatible with the possibility that MAG and Fyn act independently to initiate myelination. Hypomyelination of optic nerves was not related to a loss of oligodendrocytes, indicating that the phenotype results from impaired interactions between oligodendrocyte processes and axons and/or impaired morphological maturation of oligodendrocytes. Finally, we demonstrate that Fyn, unlike MAG, is not involved in the formation of ultrastructurally intact myelin sheaths.

Key words: double knock-out mutant; hypomyelination; Fyn; MAG; oligodendrocyte; optic nerve; spinal cord
Myelin sheaths electrically insulate axons, and the generation of action potentials is thus confined to myelin-free regions of axons, the nodes of Ranvier. The resulting saltatory conduction of nerve impulses increases the speed at which information is propagated along axons. Given the functional importance of myelin, it is of particular interest to understand the differentiation of myelinating glial cells, the interaction between myelin-forming glial cells and axons, and the formation and maintenance of myelin at the molecular level.

One of the molecules involved in the formation and maintenance of myelin is the myelin-associated glycoprotein (MAG), a member of the immunoglobulin superfamily (Arquint et al., 1987; Lai et al., 1987; Salzer et al., 1987). The analysis of MAG-deficient mice has revealed abnormal interactions between myelin-forming glial cells and axons and a variety of ultrastructural abnormalities of myelin sheaths in the CNS, and degeneration of myelin and axons in the peripheral nervous system (PNS) (Li et al., 1994; Montag et al., 1994) (for review, see Schachner and Bartsch, 2000). Of particular interest for the present study is a delayed myelination of developing optic nerves of MAG-deficient mice (Montag et al., 1994) and a hypomyelination of optic nerves of adult null mutants (Bartsch et al., 1997). Myelination in the PNS, in contrast, was not retarded in the absence of MAG (Montag et al., 1994). All these results demonstrate that MAG performs different functional roles in the CNS and PNS. Indeed, it has been demonstrated recently that the

\footnotetext{
Received March 3, 2000; revised July 6, 2000; accepted July 25, 2000.

We are grateful to Dr. Philippe Soriano for providing the Fyn-deficient mice, Dr. Klaus-Armin Nave for the pS4 plasmid, Dr. Jacqueline Trotter, Eva-Maria Krämer, Marius Ader, and Mathias Evers for critically reading this manuscript, Sandra Schmidt for help with behavioral tests, Christiane Born and Ester Gui-Xia Yu for expert technical assistance, and Kathrin Mannigel and Eva Kronberg for animal care.

Correspondence should be addressed to Udo Bartsch, Zentrum für Molekulare Neurobiologie, Universität Hamburg, Martinistraße 52, D-20246 Hamburg, Germany. E-mail: udo.bartsch@zmnh.uni-hamburg.de.

Dr. Biffiger's present address: Prionics AG, Winterthurerstraße 190, CH-8057 Zürich, Switzerland.

Dr. Montag's present address: Neurogenetics, Leibniz Institute for Neurobiology, Brenneckestra $\beta$ e 6, D-39118 Magdeburg, Germany

Copyright (C) 2000 Society for Neuroscience $0270-6474 / 00 / 207430-08 \$ 15.00 / 0$
}

"large" MAG isoform (L-MAG) is the functionally important isoform in the CNS, whereas the "small" MAG isoform (S-MAG) is sufficient to maintain myelin and axons in the PNS (Fujita et al., 1998).

The elucidation of signal transduction mechanisms is critical to understand the differential action of the L-MAG and S-MAG isoforms during development and maintenance of CNS and PNS myelin. The nonreceptor-type tyrosine kinase Fyn, a member of the Src family, has been identified as a signaling molecule downstream of L-MAG (Umemori et al., 1994). MAG and Fyn are coexpressed by oligodendrocytes, coimmunoprecipitation experiments revealed an association of Fyn with MAG, and cross-linking of MAG with antibodies stimulated Fyn kinase activity in COS cells cotransfected with L-MAG and Fyn but not in cells cotransfected with S-MAG and Fyn (Umemori et al., 1994). During early stages of myelination, L-MAG is the predominant MAG isoform in the CNS (Tropak et al., 1988; Pedraza et al., 1991), and Fyn shows highest kinase activity (Umemori et al., 1994). These data suggest that Fyn is critically involved in initial events of myelin formation. In fact, analysis of the CNS of adult Fyn-deficient mice revealed a reduction in the amount of myelin by $\sim 50 \%$ when compared with age-matched wild-type mice (Umemori et al., 1994). Given that several morphological abnormalities of myelin sheaths in MAG null mutants are related to the lack of L-MAG (Fujita et al., 1998), Fyn might also be involved in the formation of morphologically intact myelin sheaths.

To investigate the proposed functional relationship between MAG and Fyn in the process of myelination, we performed an ultrastructural analysis of optic nerves of MAG-, Fyn-, and MAG/ Fyn-deficient mice. Hypomyelination was moderate in MAG mutants, significant in Fyn mutants, and massive in MAG/Fyn double knock-out mice. These data demonstrate that the MAG-Fyn signaling pathway is complex. However, results are also compatible with the possibility that MAG and Fyn act independently to initiate myelination. Hypomyelination of the optic nerve was not related to a loss of oligodendrocytes, indicating that the phenotype might result from impaired oligodendrocyte-axon interactions or an impaired morphological maturation of oligodendrocytes. 

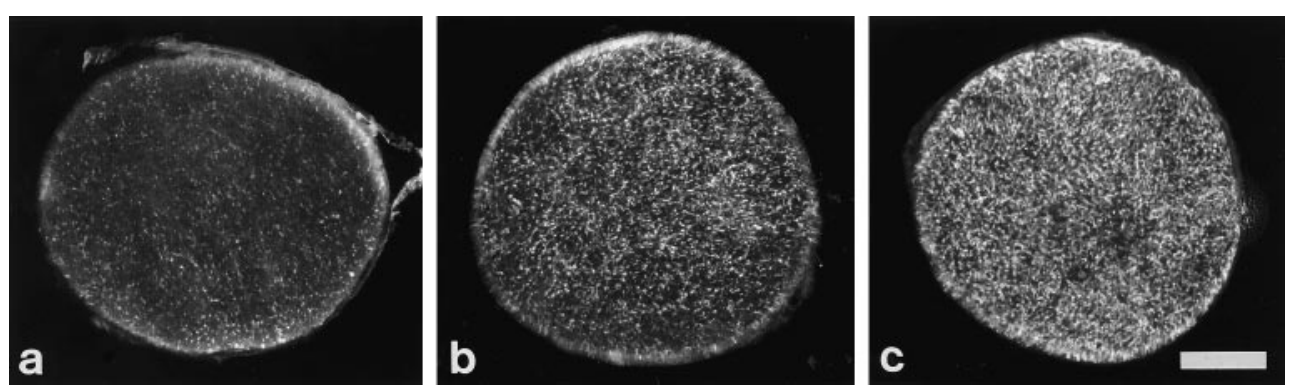

Figure 1. Visualization of unmyelinated axons in optic nerves of different genotypes. Unmyelinated retinal ganglion cell axons were visualized in optic nerves of 7-month-old wild-type $(a)$ and age-matched Fyn- $(b)$ and MAG/Fyn- $(c)$ deficient mice by immunohistochemistry using polyclonal antibodies to the neural adhesion molecule L1. Only a few L1-positive unmyelinated axons are detectable in optic nerves of wild-type mice $(a)$, whereas they are numerous in nerves of Fyn-deficient animals $(b)$. Note that the density of L1-positive axons is significantly increased in mice lacking both MAG and Fyn (c) when compared with Fyn single mutants (compare $c$ with $b$ ). Scale bar (in $c$ ): $a-c, 100 \mu \mathrm{m}$.

\section{MATERIALS AND METHODS}

Animals. Generation of mice deficient in MAG or Fyn has been described previously (Stein et al., 1992; Montag et al., 1994). Double knock-out mutants deficient in both MAG and Fyn were obtained by cross-breeding the corresponding single mutants. The genotypes of animals were determined by Southern blot analysis of DNA from tail biopsies (Stein et al. 1992; Montag et al., 1994).

Immunohistochemistry. Immunohistochemical analysis of optic nerves was performed as described previously (Bartsch et al., 1989). In brief, optic nerves were quickly removed, embedded in methylcellulose, and frozen in methylbutane cooled by liquid nitrogen. Cross sections of optic nerves, 14 $\mu \mathrm{m}$ in thickness, were prepared and incubated with polyclonal L1 antibodies to visualize unmyelinated retinal ganglion cell axons (Bartsch et al., 1989). Primary antibodies were detected with fluorescein isothiocyanateconjugated antibodies (Dianova, Hamburg, Germany), and sections were analyzed using an Axiophot microscope (Zeiss, Oberkochen, Germany).

In situ hybridization. A digoxigenin-labeled cRNA probe recognizing the transcripts of the two products of the PLP/DM-20 gene, proteolipid protein (PLP) and its smaller isoform DM-20, was prepared from plasmid pS4 containing a $1.4 \mathrm{~kb}$ Bam HI-PstI fragment of plasmid C4 (Nave et al., 1986) recloned into pSP72 (Promega, Mannheim, Germany) (Laeng et al, 1996). The PLP/DM-20 probe was used to visualize cells of the oligodendrocyte lineage in longitudinally sectioned optic nerves of wild-type mice and the different mutants (all 7-month-old). We also generated a probe specifically recognizing PLP transcripts to visualize differentiated oligodendrocytes. To this aim, the $105 \mathrm{bp}$ PLP-specific sequence contained in plasmid $\mathrm{pS} 4$ was amplified by PCR using $5^{\prime}$-specific primer PLP forward (TTCTCGAGTAACAGGGGGCCAGAAG) and $33^{\prime}$-specific primer PLP backward (TGAAGCTTGTCGGGATGTCCTAGCC) introducing Xho and HindIII restriction sites into the product. PCR was performed in a Perkin-Elmer Gene Amp PCR System 9700 under the following conditions. Fifty nanograms of PstI-linearized plasmid pS4, 100 pmol of each primer, $1.5 \mathrm{U}$ of Taq-DNA polymerase (Promega), and $0.25 \mathrm{~mm}$ each dNTP in $50 \mu l$ of $1 \times$ reaction buffer (Promega) were denatured for $3 \mathrm{~min}$ at $94^{\circ} \mathrm{C}$, followed by $40 \mathrm{cycles}$ at $94^{\circ} \mathrm{C}$ for $30 \mathrm{sec}, 50^{\circ} \mathrm{C}$ for $30 \mathrm{sec}$, and $72^{\circ} \mathrm{C}$ for $1 \mathrm{~min}$. PCR products were separated on a $2 \%$ agarose gel, and the 117 bp DNA fragment was purified with Qiaex (Qiagen, Hilden, Germany). After restriction with XhoI and HindIII, the fragment was cloned into pBluescript KS - (Stratagene, Heidelberg, Germany). One correct clone (pDM-PLP.2) was used for generation of sense (linearized with HindIII, T3 RNA polymerase) and antisense (linearized with XhoI, T7 RNA polymerase) PLP-specific probes. Generation of a MAG-specific cRNA probe has been described previously (Bartsch et al., 1994).

In situ hybridization analysis was performed as described previously (Bartsch et al., 1992). After hybridization and washing, sections were incubated with alkaline phosphatase-conjugated antibodies to digoxigenin (Roche Diagnostics, Mannheim, Germany) and developed using 4-nitroblue tetrazolium chloride and 5-bromo-4-chloro-3-indolylphosphate (both from Sigma, Deisenhofen, Germany). In each experiment, sections from all different genotypes were processed in parallel, and all sections hybridized with the same probe were developed for the same period of time.

The number of cells labeled with the PLP-specific probe was determined in cross sections (14- $\mu \mathrm{m}$-thick) of optic nerves (middle region of the nerve) of wild-type and MAG/Fyn-deficient mice (all between 4- and 6-monthold) using the Neurolucida image analysis system. All PLP-positive cells or cell fragments per sections were counted. Six sections per animal and six animals per genotype were analyzed. Consecutive cross sections from wild-type nerves were additionally hybridized with the MAG antisense probe, and the number of MAG-positive cells was determined as described for PLP-positive cells. Statistical analysis of data were performed using the unpaired $t$ test.

In all experiments, specificity of labeling was controlled by hybridizing some sections with the corresponding sense probes and by hybridizing the oligodendrocyte-free retinal end of wild-type and mutant optic nerves with
PLP/DM-20-, PLP-, or MAG-specific antisense probes. Labeled cells were not observed in any of these experiments.

Electron microscopy. Animals were fixed by perfusion using $4 \%$ paraformaldehyde and $2.5 \%$ glutaraldehyde in PBS, pH 7.3. Optic nerves and spinal cords were quickly removed and immersed in $2 \% \mathrm{OsO}_{4}$ for $2 \mathrm{hr}$ at room temperature. Subsequently, tissue was dehydrated in an ascending series of methanol and embedded in Epon resin. Ultrathin sections from middle regions of optic nerves and cervical levels of spinal cords were counterstained with lead citrate and analyzed using an EM 10 (Zeiss).

Quantitative analysis. The percentage of unmyelinated axons was determined in ultrathin sections of optic nerves from 4-month-old wild-type and MAG-deficient mice, and 2-, 4- and 9-month-old Fyn and MAG/Fyn knock-out mice. Randomly selected regions of optic nerves were photographed at a magnification of $10,000 \times$. Myelinated and unmyelinated ganglion cell axons visible with their entire circumference on the photomicrographs were counted at a final magnification of $28,000 \times$, and the percentage of unmyelinated axons was calculated (Bartsch et al., 1997). Between four and six animals and 615 and 2918 axons (depending on the degree of hypomyelination) per animal were analyzed for each developmental age and genotype. Values for 2- and 9-month-old wild-type and MAG-deficient mice were determined in the same way and were taken from Bartsch et al. (1997).

The ultrastructure of myelin sheaths was studied in optic nerves of 2- and 9-month-old wild-type and mutant mice. Myelinated retinal ganglion cell axons were randomly selected, and the percentage of myelin sheaths with a periaxonal cytoplasmic collar spanning less than half of the axonal circumference was determined. We also determined the percentage of multiply myelinated axons, myelin sheaths with noncompacted regions of myelin, myelin sheaths forming redundant myelin, and myelin sheaths with intramyelinic or periaxonal signs of degeneration. Myelin sheaths with such defects were defined as "affected myelin sheaths." Four animals and between 304 and 422 axons per animal were analyzed for each developmental age and genotype. Statistical analysis of data were performed using the unpaired $t$ test.

\section{RESULTS}

\section{The MAG/Fyn-deficient mouse}

Mice deficient in MAG and Fyn were generated by cross-breeding the respective single mutants (Stein et al., 1992; Montag et al., 1994). MAG/Fyn double mutants were fertile and survived for at least 1 year. When number-coded age-matched wild-type and MAG/Fyn-deficient males (nine wild-types and 11 MAG/Fyn mutants between 4 and 6 months old) were observed in their cages for a 5 min period, obvious behavioral differences were not apparent between genotypes. When the same animals were placed on a smooth platform and observed for 2 min, very weak shivering for short time periods was noticed for four animals. All of these animals were MAG/Fyn double mutants. When animals were repeatedly lifted up at their tails and placed back onto the platform (10 times over a period of $10-15 \mathrm{sec}$ ), all MAG/Fyn mutants, but none of the wild-type controls, displayed prominent and shortlasting tremors that could be reinduced by slightly touching the animals.

\section{Density of L1-immunoreactive axons in optic nerves of different genotypes}

Myelin-associated glycoprotein and Fyn tyrosine kinase are both involved in the initiation of myelination in the CNS (Montag et al., 1994; Umemori et al., 1994; Bartsch et al., 1997). In the optic nerve 


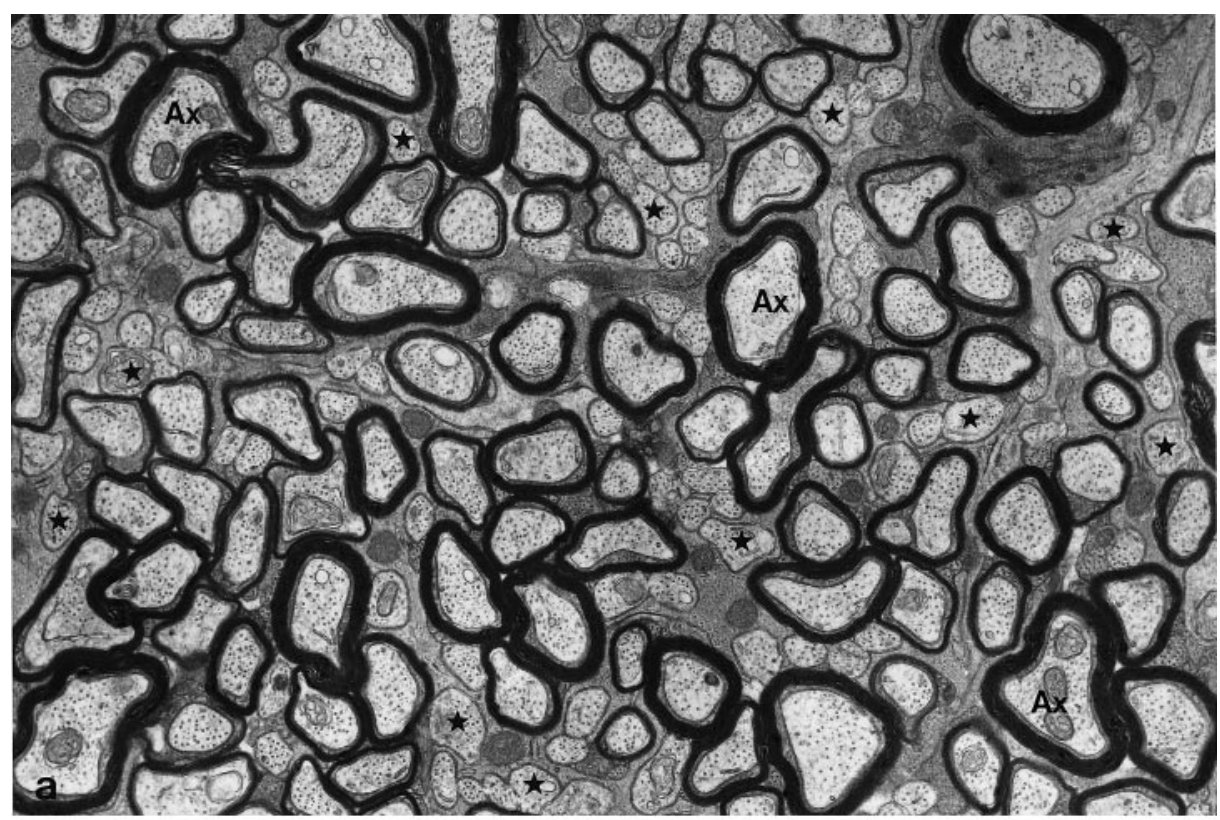

Figure 2. Ultrastructure of optic nerves of Fyn- and MAG/Fyn-deficient mice. Optic nerves of 2-month-old Fyn-deficient mice $(a)$ contain a high number of small-sized unmyelinated axons (some labeled with asterisks). Compared with Fyn mutants, a significantly increased number of unmyelinated axons (some labeled with asterisks) is present in optic nerves of age-matched MAG/Fyn double knock-out mice $(b)$. Some myelin sheaths in MAG/Fyn double mutants form redundant myelin (white dots in $b$ ) or contain noncompacted regions of myelin (curved arrows in $b$ ). Ax, Axon. Scale bar (in $b$ ): $a, b, 1 \mu \mathrm{m}$.

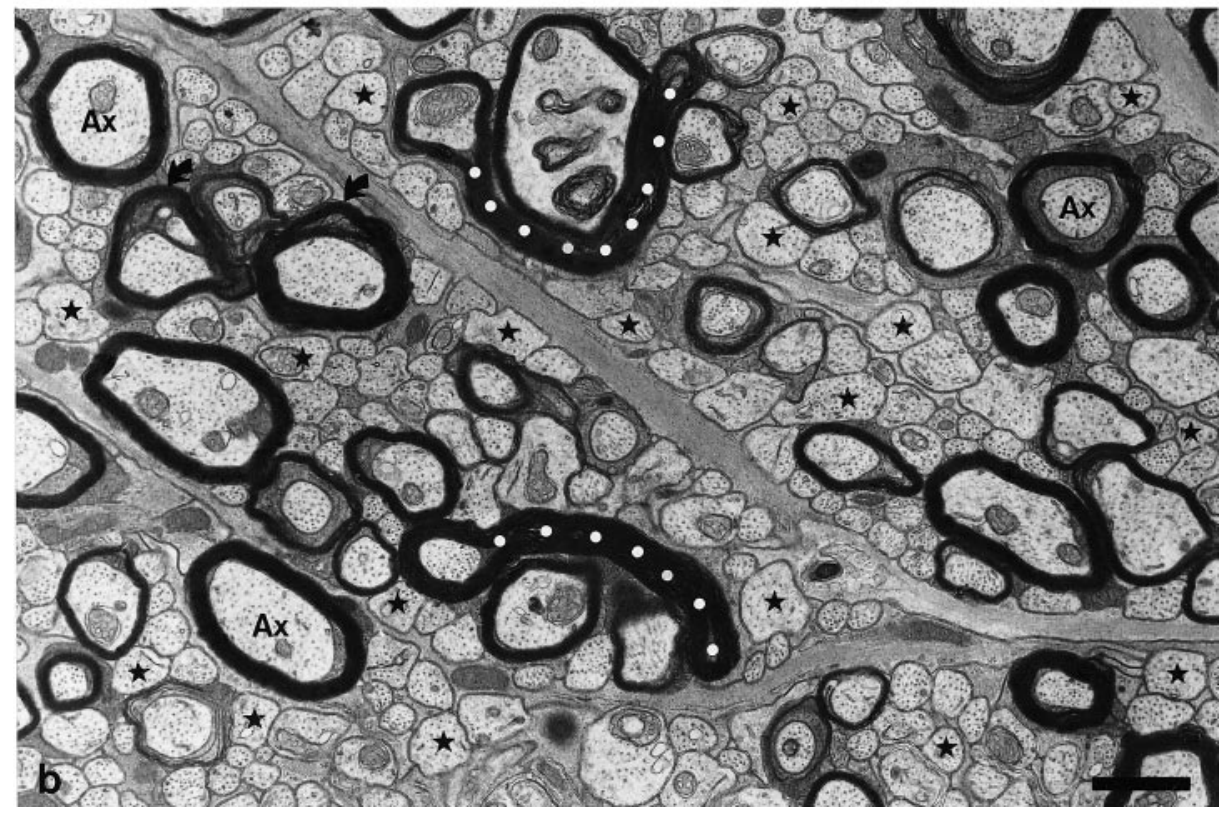

of mice, the neural adhesion molecule L1 is present on unmyelinated axons but is absent from myelinated axons (Bartsch et al., 1989). Cross sections of optic nerves from 7-month-old wild-type mice and age-matched MAG-, Fyn-, and MAG/Fyn-deficient animals were therefore incubated with polyclonal anti-L1 antibodies to visualize unmyelinated axons at the light microscopic level (Fig. 1). Only a few L1-positive axons were detectable in wild-type nerves (Fig. 1a), reflecting the fact that the vast majority of retinal ganglion cell axons in the adult mouse optic nerve is myelinated. Compared with wild-type mice, the density of L1-positive axons increased slightly in MAG-deficient mice (data not shown), significantly in Fyn mutants (Fig. 1b), and dramatically in MAG/Fyn double mutants (Fig. 1c).

\section{Qualitative ultrastructural analysis of optic nerves}

The majority of axons in the optic nerve of 2-month-old wild-type mice was surrounded by a myelin sheath, and only a few unmyelinated axons were detectable. The number of unmyelinated axons was significantly increased in age-matched MAG mutants. Unmyelinated axons in MAG mutants were all of small caliber, and their number decreased in 4- and 9-month-old MAG-deficient mice (data not shown) (Bartsch et al., 1997; see below). The density of unmyelinated axons in 2-month-old Fyn-deficient mice (Fig. 2a) was significantly increased when compared with age-matched MAG mutants. A high number of unmyelinated axons was still present in 9-month-old Fyn mutants, the oldest animals analyzed (data not shown; see below). Similar to MAG-deficient mice, unmyelinated axons in Fyn mutants were of small caliber at all developmental ages studied. In 2-month-old MAG/Fyn double knock-out mice (Fig. $2 b$ ), the number of unmyelinated axons was dramatically increased when compared with Fyn mutants of similar age. Many unmyelinated small-sized axons were in direct contact with each other, without interdigitating glial processes (Fig. 2b). Similar results were obtained for 4- and 9-month-old MAG/Fyn double mutants (data not shown; see below).

To obtain information about the phenotype of MAG/Fyn double mutants in other CNS regions, we studied the cervical spinal cord of 2-month-old MAG/Fyn double mutants and age-matched wild-type mice (six animals for each genotype). Interestingly, these studies revealed a different degree of hypomyelination in different regions of the cord. The corticospinal tract of the double mutant was massively hypomyelinated (data not shown), and the degree of hypomyelination was reminiscent of that observed in the optic 


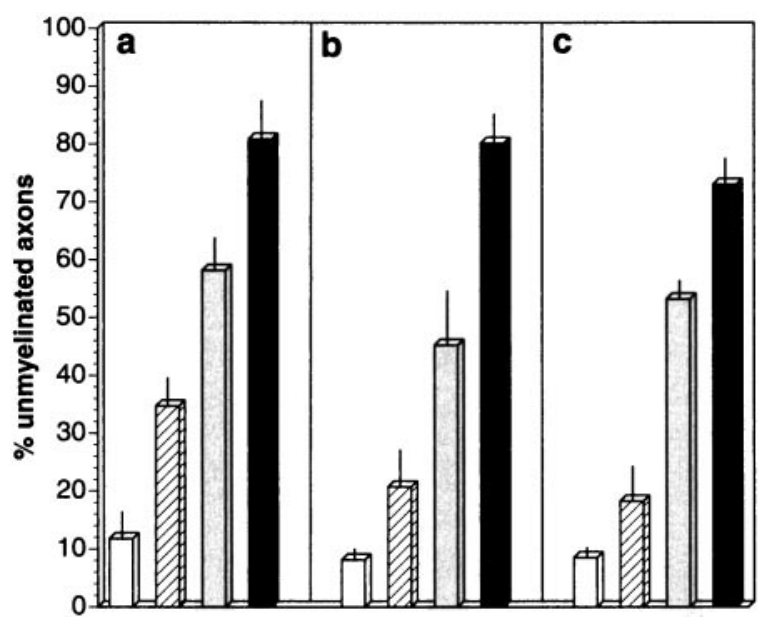

Figure 3. The percentage of unmyelinated axons in the optic nerve of adult mice of different genotypes. The percentage of unmyelinated axons from the total axon population was determined in optic nerves of 2- $(a)$, 4(b), and 9- (c) month-old wild-type (open bars), MAG- (hatched bars), Fyn(stippled bars), and MAG/Fyn- (filled bars) deficient mice. Bars for 4-month-old wild-type and MAG-deficient mice represent the mean value ( \pm SD) of five and four animals, respectively. Bars for all other genotypes and developmental ages represent the mean value $( \pm S D)$ of six animals. Note that the number of unmyelinated axons is significantly increased in optic nerves of MAG/Fyn double mutants when compared with Fyn single mutants $(p<0.0001$ according to the unpaired $t$ test)

nerve. Hypomyelination was also apparent in other regions of the cord (i.e., fasciculus cuneatus and gracilis or ventral funiculus; data not shown) but was significantly less pronounced than in the optic nerve or corticospinal tract. Thus, fiber tracts containing relatively small-sized myelinated axons were severely hypomyelinated, whereas fiber tracts containing large-sized axons were much less affected.

\section{Quantification of unmyelinated axons in optic nerves of different genotypes}

The percentage of unmyelinated axons from the entire axon population was determined in optic nerves of 4-month-old wild-type and MAG-deficient mice and 2-, 4-, and 9-month-old Fyn- and MAG/Fyn-deficient mice. Values for 2- and 9-month-old wild-types and MAG mutants were taken from Bartsch et al. (1997). Except for 4-month-old wild-type $(n=5)$ and MAG-deficient $(n=4)$ mice, six animals were analyzed for each genotype and developmental age (Fig. 3).

As reported previously, $11.7 \pm 4.3 \%$ (mean $\pm \mathrm{SD}$ ) of all axons are unmyelinated in the optic nerve of 2-month-old wild-type mice (Bartsch et al., 1997) (Fig. 3). A similar percentage of unmyelinated axons was found in $4-(8.1 \pm 1.8 \%)$ and $9-(8.5 \pm 1.8 \%)$ month-old wild-type animals (Fig. 3). A significantly increased number of unmyelinated axons was present in optic nerves of 2-month-old MAG mutants $(34.7 \pm 5.5 \%)$, which decreased with increasing age (20.7 \pm 6.6 and $18.2 \pm 6.0 \%$ for 4 - and 9-month-old MAG mutants, respectively) (Bartsch et al., 1997; present study). Compared with 2-month-old MAG mutants, a significantly higher percentage of axons was unmyelinated in the optic nerve of age-matched Fyn mutants $(58.2 \pm 5.7 \% ; p<0.0001$ according to the unpaired $t$ test $)$, and this value did not change significantly with increasing age $(45.2 \pm 9.3 \%$ for 4-month-old, and $53.2 \pm 3.2 \%$ for 9 -month-old Fyn mutants) (Fig. 3). Finally, a severe hypomyelination was observed in the optic nerve of MAG/Fyn double knock-out mutants. The vast majority of ganglion cell axons $(80.8 \pm 6.3 \%)$ was unmyelinated in optic nerves of 2-month-old double mutants. A similar degree of hypomyelination was observed in the optic nerve of 4$(80.1 \pm 5.6 \%)$ and $9-(74.0 \pm 4.2 \%)$ month-old MAG/Fyn-deficient mice (Fig. 3). Values for Fyn- and MAG/Fyn-deficient mice were significantly different from each other at all ages analyzed $(p<$ $0.0001)$.

\section{Visualization of oligodendrocytes in optic nerves of different genotypes}

Loss of oligodendrocytes might cause hypomyelination in the optic nerve of different mutants. To investigate this possibility, longitudinal sections of optic nerves from different genotypes (all 7-monthold) were subjected to in situ hybridization analysis using a PLP/ DM-20 cRNA probe. PLP/DM-20-positive cells were restricted to the distal myelinated part of the nerve and were absent from the unmyelinated retinal end of the nerve and from the retina (Fig. 4). Significant differences in the number of PLP/DM-20-positive cells between nerves of wild-type, MAG-, Fyn-, and MAG/Fyndeficient mice were not apparent (Fig. 4, compare $a, b$ ). There were also no apparent differences in the staining intensity between the different genotypes. No labeled cells were found in sections incubated with the corresponding sense probe (data not shown).

Because the DM-20 isoform is expressed early during oligodendrocyte development (Timsit et al., 1995; Fanarraga et al., 1996), the PLP/DM-20 probe might have labeled immature cells of the

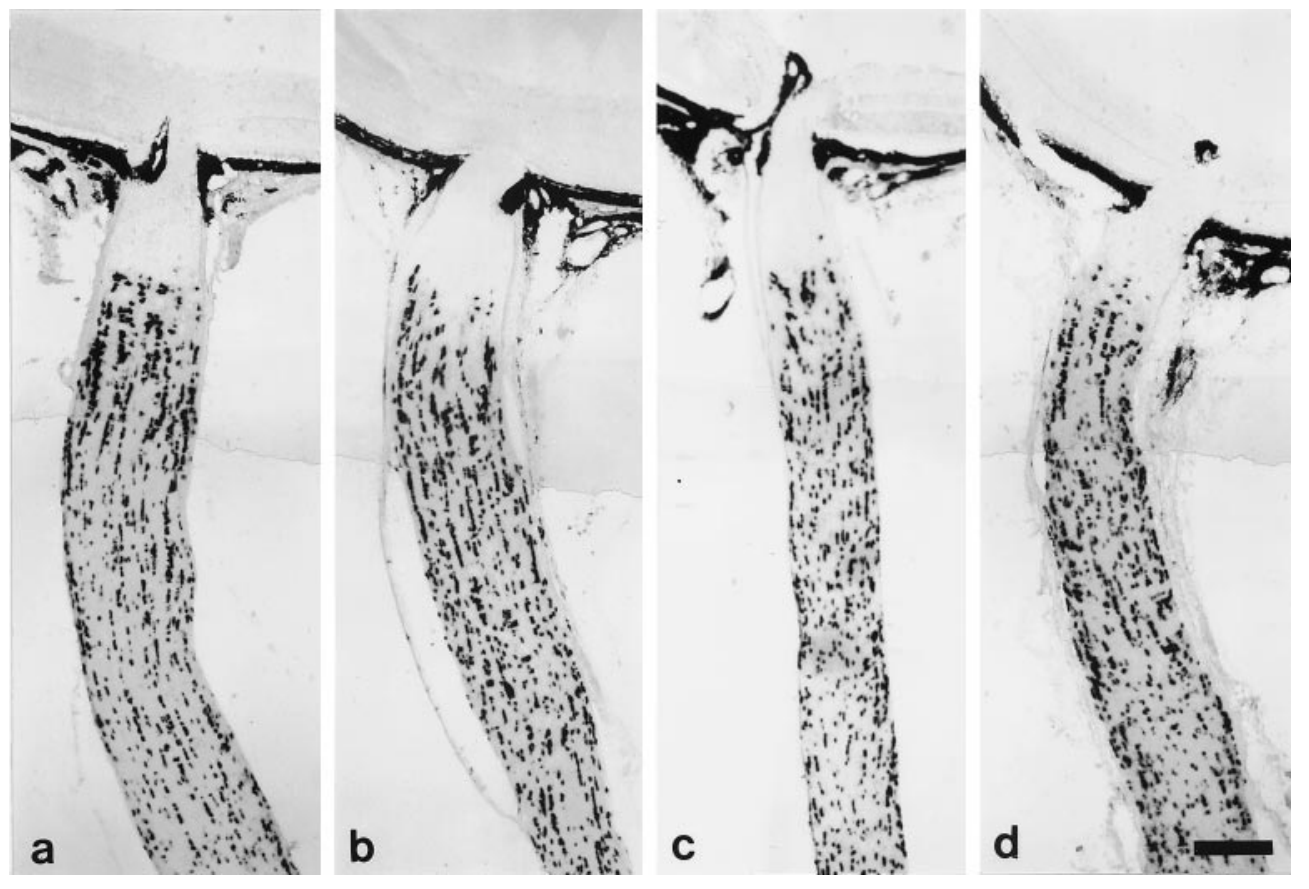

Figure 4. Visualization of PLP/DM-20positive cells in optic nerves of different genotypes. PLP/DM-20-positive cells were visualized in optic nerves of 7-month-old wild-type $(a)$, MAG- $(b)$, Fyn- $(c)$, and MAG/Fyn- $(d)$ deficient mice by in situ hybridization. Note the similar density and labeling intensity of positive cells in optic nerves of different genotypes. Scale bar (in $d): a-d, 200 \mu \mathrm{m}$. 


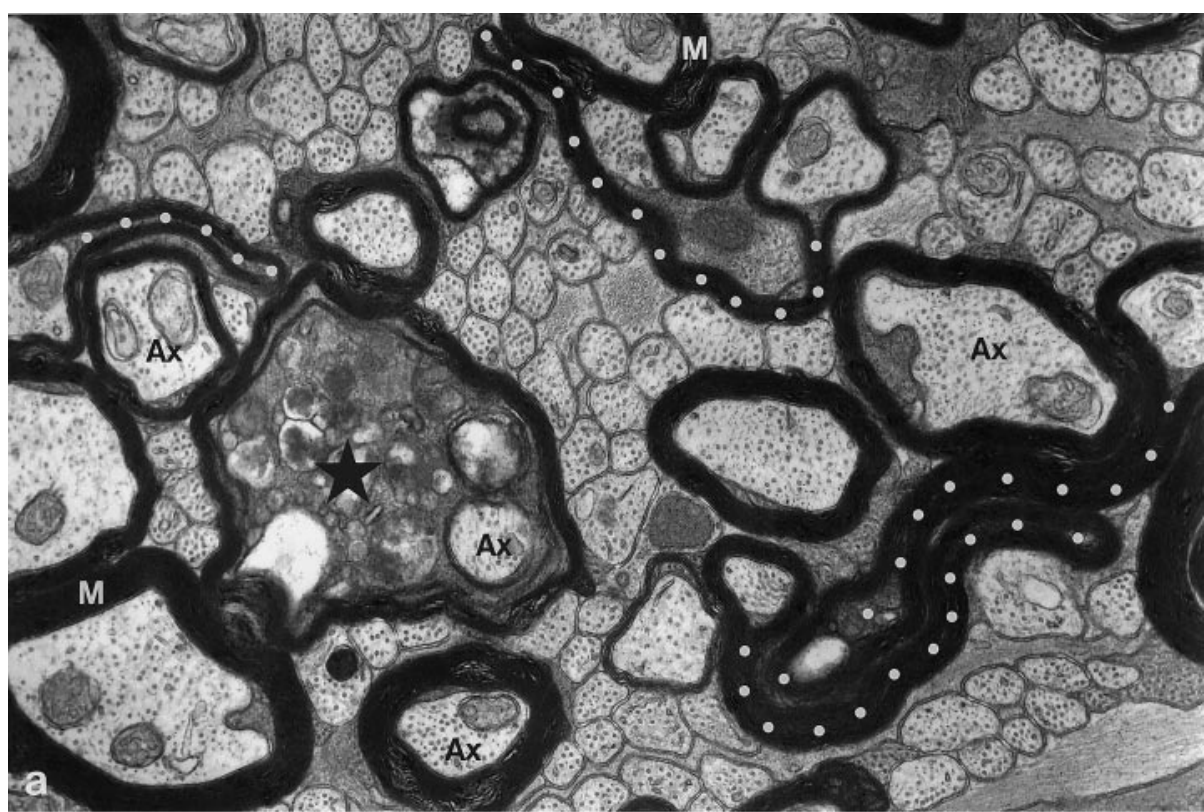

Figure 5. Ultrastructure of myelin sheaths in optic nerves of Fyn- and MAG/Fyn-deficient mice. Most myelin sheaths in optic nerves of 2-monthold MAG/Fyn mutants $(a)$ lack a well developed periaxonal cytoplasmic collar, and some sheaths form redundant myelin coursing away from the axon (white dots in $a$ ) or show degenerative alterations (asterisk in $a$ ). Myelin sheaths in optic nerves of age-matched Fyn mutants $(b)$, in contrast, appear morphologically intact. Note the well developed periaxonal cytoplasmic collar (arrowheads in $b$ ) of most sheaths. $A x$, Axon; $M$, myelin. Scale bar (in $b$ ): $a, 0.5 \mu \mathrm{m} ; b, 0.25 \mu \mathrm{m}$.

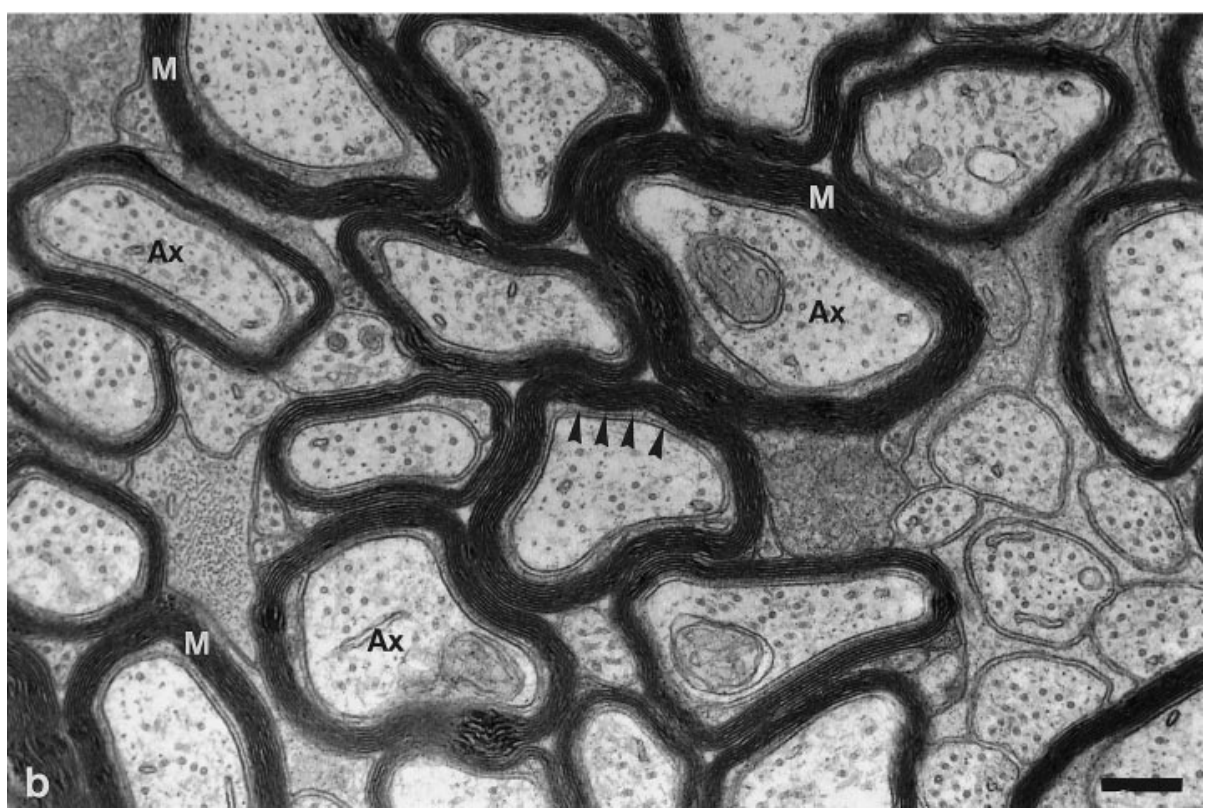

oligodendrocyte cell lineage in mutant nerves. Therefore, we hybridized cross sections from the middle part of optic nerves from adult wild-type and age-matched MAG/Fyn mutants with a probe specifically recognizing PLP transcripts. Again, the number of labeled cells and the intensity of the in situ hybridization signal was not obviously different between genotypes (data not shown). As a next step, we counted the number of PLP-positive cells in numbercoded cross sections of wild-type and MAG/Fyn-deficient nerves. Six animals of each genotype and six sections per animal were analyzed. The number of PLP-positive cells (and cell fragments) per cross section of wild-type nerves was $119.3 \pm 13.3$ (mean \pm SD). Consecutive sections of wild-type nerves were also hybridized with the MAG probe and contained almost identical numbers of labeled cells $(122.1 \pm 11.0)$. The number of PLP-positive cells per cross section of MAG/Fyn-deficient nerves was $130.2 \pm 11.8$, a value not significantly different from that determined for PLP-positive cells in wild-type nerves ( $p>0.15$ according to the unpaired $t$ test).

\section{Qualitative and quantitative ultrastructural analysis of myelin sheaths}

Myelin sheaths in the CNS of MAG knock-out mice show a variety of morphological defects. Most sheaths lack a well developed peri- axonal cytoplasmic collar and some contain noncompacted regions of myelin, form redundant myelin, or show intramyelinic or periaxonal signs of degeneration (for review, see Bartsch, 1996). Most of these morphological defects were also found in mouse mutants deficient in the L-MAG isoform (Fujita et al., 1998). We therefore analyzed whether Fyn, proposed to act as a signaling molecule downstream of L-MAG (Umemori et al., 1994), is involved in the formation of morphologically intact myelin sheaths.

Ultrastructural abnormalities of myelin sheaths in the optic nerve and cervical spinal cord of MAG/Fyn double mutants were similar to those found in MAG null mutants. The majority of myelin sheaths in the double mutant lacked a well developed periaxonal cytoplasmic collar, and some sheaths formed redundant myelin coursing away from the axon (Figs. $2 b, 5 a$ ). Myelin sheaths with periaxonal (data not shown) or intramyelinic (Fig. 5a) degenerative alterations or noncompacted regions of myelin (Fig. 2b) were also present in MAG/Fyn-deficient nerves. Importantly, morphological defects different from those characteristic for MAGdeficient mice were not observed in the double mutant. Whereas morphologically abnormal myelin sheaths were frequently observed in MAG/Fyn double mutants (see below), they were absent 

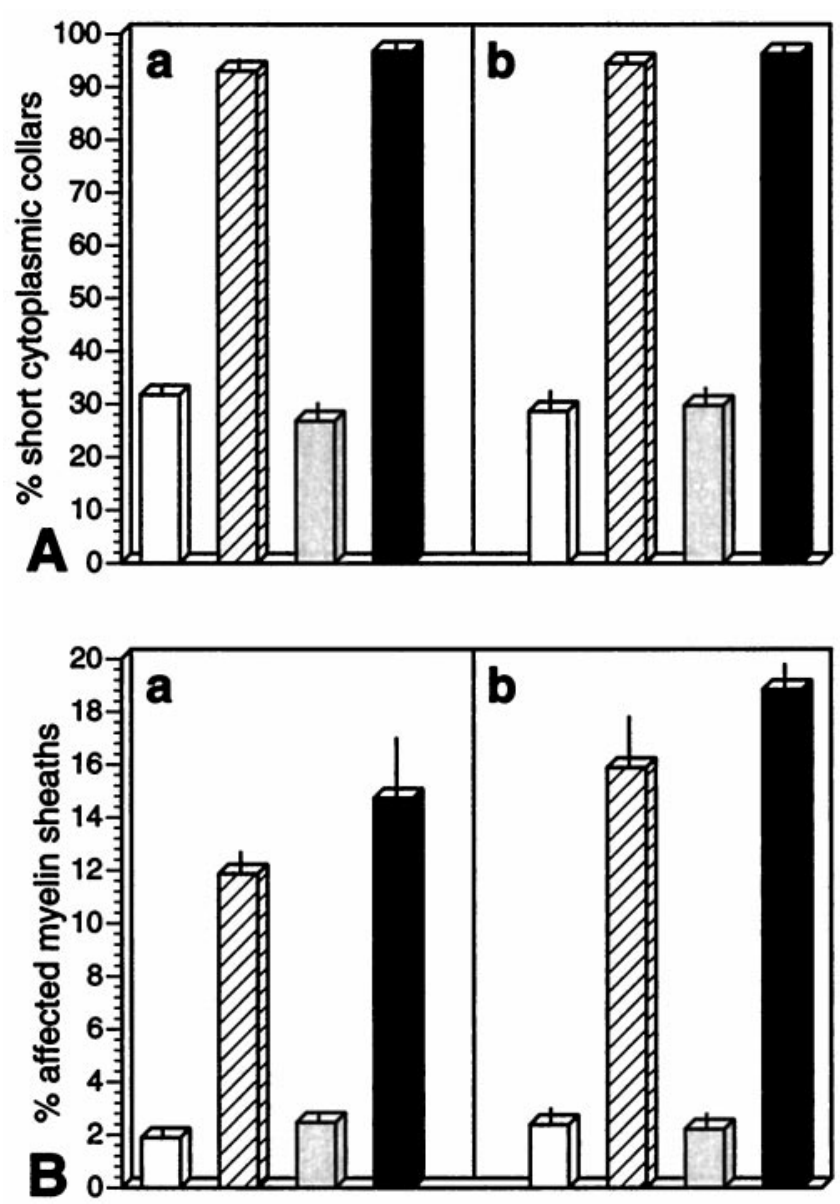

Figure 6. Frequency of morphologically affected myelin sheaths in different genotypes. $A$, The percentage of myelin sheaths with an oligodendrocyte periaxonal cytoplasmic collar spanning less than half of the axonal circumference was determined in optic nerves of 2- $(a)$ and 9-month-old $(b)$ wild-type (open bars) and MAG- (hatched bars), Fyn- (stippled bars), and $\mathrm{MAG} /$ Fyn-deficient ( filled bars) mice. Each bar represents the mean value $( \pm \mathrm{SD})$ of four animals. Note that the formation of well developed periaxonal cytoplasmic collars is dependent on the presence of MAG but not on the presence of Fyn. $B$, The frequency of multiply myelinated axons, myelin sheaths with regions of noncompacted myelin, myelin sheaths forming redundant myelin, and myelin sheaths with signs of intramyelinic or periaxonal degeneration was determined in optic nerves of 2- $(a)$ and 9-monthold (b) wild-type (open bars), MAG- (hatched bars), Fyn- (stippled bars), and MAG/Fyn-deficient ( filled bars) mice. Each bar represents the mean value $( \pm \mathrm{SD})$ of four animals. Note that Fyn is not involved in the formation of ultrastructurally intact myelin sheaths.

from optic nerves of age-matched Fyn-deficient mice. For instance, most myelin sheaths in Fyn mutants contained a well developed periaxonal cytoplasmic collar (Fig. $5 b$ ). In fact, qualitative analysis revealed no obvious differences in the ultrastructure of myelin sheaths between Fyn mutants (Fig. $5 b$ ) and age-matched wild-type mice (data not shown).

Quantitative analysis revealed that $\sim 30 \%$ of myelin sheaths of 2and 9-month-old wild-type mice contained a periaxonal cytoplasmic collar that spans less than half of the axonal circumference. In comparison, almost every myelin sheath (i.e., 95\%) lacked a well developed cytoplasmic collar in age-matched MAG mutants (Fig. $6 A)$. These observations are in agreement with previous results (Montag et al., 1994). In Fyn mutants, in contrast, the percentage of myelin sheaths with periaxonal collars spanning less than half of the axonal circumference was similar to that observed in wild-type mice at all ages analyzed. Moreover, there was no significant difference in the percentage of myelin sheaths with short periaxonal cytoplasmic collars between MAG and MAG/Fyn mutants (Fig. 6A). We next determined the frequency of multiply myelinated axons, myelin sheaths with noncompacted regions of myelin, myelin sheaths forming redundant myelin, and myelin sheaths showing intramyelinic or periaxonal degeneration in the different genotypes (Fig. 6B). In optic nerves of wild-type mice, such defects were only rarely observed, and only $1.9 \pm 0.3$ (mean \pm SD) and $2.4 \pm 0.5 \%$ of all myelin sheaths were morphologically affected in 2- and 9-month-old animals, respectively. Optic nerves of MAG mutants, in comparison, contained significantly more morphologically abnormal myelin sheaths $(11.9 \pm 0.8$ and $15.9 \pm 1.9 \%$ for 2 and 9-month-old MAG mutants, respectively). The percentage of morphologically abnormal myelin sheaths in Fyn mutants was similar to that observed in wild-type mice $(2.5 \pm 0.2 \%$ for 2 -month-old and $2.2 \pm 0.4 \%$ for 9-month-old Fyn mutants), and the percentage of affected sheaths in $\mathrm{MAG} / \mathrm{Fyn}$ double mutants was similar to that observed in MAG mutants $(14.7 \pm 2.3$ and $18.8 \pm 0.8 \%$ for 2 - and 9-month-old double mutants, respectively) (Fig. 6B). Thus, formation of ultrastructurally intact myelin sheaths is not dependent on the presence of Fyn.

\section{DISCUSSION}

Initiation of myelination in the CNS of MAG-deficient mice is impaired, as indicated by a reduced number of myelin sheaths in optic nerves of young postnatal MAG mutants (Montag et al., 1994) and a significantly increased number of small-sized unmyelinated axons in optic nerves of young adult null mutants (Bartsch et al., 1997; this study). Fyn, a nonreceptor-type tyrosine kinase, has been identified as a signaling molecule downstream of L-MAG and has also been implicated in initiation of myelination. In fact, the amount of myelin in brains of independently generated Fyndeficient mice was reduced by $\sim 40-50 \%$ when compared with age-matched wild-type animals (Umemori et al., 1994). Our quantitative ultrastructural analysis of Fyn-deficient optic nerves is in good agreement with these data and supports a critical role of Fyn during initiation of myelination in the CNS; $\sim 50 \%$ of all axons were unmyelinated in optic nerves of adult Fyn mutants compared with only $\sim 10 \%$ unmyelinated axons in wild-type mice. Remarkably, hypomyelination of the optic nerve of MAG/Fyn-deficient mice was significantly increased when compared with Fyn knockout mice, with $\sim 80 \%$ of all axons being unmyelinated in the double mutant. The small caliber of unmyelinated axons in all different mutants indicates that reduced amounts of myelin result from impaired initiation of myelination rather than from demyelination. This notion is supported by the finding that formation of myelin sheaths in optic nerves of 10-d-old MAG- (Montag et al., 1994), Fyn-, and MAG/Fyn-deficient mice was significantly delayed when compared with age-matched wild-type mice (our unpublished observations). Severe hypomyelination in the double mutant demonstrates that the MAG-Fyn signaling pathway is of prime importance for the initiation of myelination in the CNS. Our data also suggest that this signaling pathway is complex and are indicative for compensatory mechanisms in the respective single mutants. Alternatively, MAG and Fyn might initiate myelination independently from each other, and the severe phenotype of MAG/Fyn double mutant mice might result from a combination of phenotypes of the respective single mutants.

Hypomyelination of the optic nerve of Fyn mutants was significantly increased when compared with MAG mutants. This observation suggests the presence of MAG-independent signaling pathways of Fyn, ultimately resulting in the initiation of myelination. It is interesting in this respect that high Fyn kinase activity has been observed in differentiating oligodendrocyte progenitor cultures before the expression of MAG (Osterhout et al., 1999). The glycosylphosphatidylinositol (GPI)-anchored $120 \mathrm{kDa}$ isoform of the neural cell adhesion molecule (NCAM120) and the recognition molecule F3/F11/contactin are expressed by immature and differentiated cells of the oligodendrocyte lineage (Bhat and Silberberg, 1986; Trotter et al., 1989; Koch et al., 1997). Interestingly, both GPI-linked recognition molecules have been demonstrated recently to be associated with Fyn in detergent-insoluble glycosphingolipid-rich microdomains (DIGs) of oligodendrocytes and myelin (Krämer et al., 1999). Kinase activity in DIGs isolated from oligodendrocytes and myelin 
was maximal at stages of active myelination, and antibody-mediated cross-linking of F3/F11/contactin in the oligodendrocyte cell line Oli-neu stimulated Fyn kinase activity specifically in DIGs (Krämer et al., 1999). Thus, interaction of NCAM120 and F3/F11/contactin with axonal ligands might initiate myelination by stimulating Fyn kinase activity in oligodendrocytes, possibly via transmembrane linker proteins. However, electron microscopic investigations of the CNS of NCAM null mutants (Cremer et al., 1994) revealed a normal formation and morphology of myelin sheaths (Schachner and Bartsch, 2000). In contrast, preliminary investigations revealed the presence of abnormal myelin profiles in the CNS of mice deficient in the recognition molecule F3/F11/contactin (Berglund et al., 1999). It will be interesting to study initiation of myelination in this null mutant. Together, the demonstration of MAGindependent signaling pathways of Fyn in oligodendrocytes is in line with our in vivo observations; hypomyelination in Fyn-deficient mice is more pronounced than in MAG mutants.

Interestingly, the degree of hypomyelination in optic nerves of MAG/Fyn double mutants was significantly increased when compared with MAG or Fyn single mutants. Approximately $80 \%$ of all retinal ganglion cell axons were unmyelinated in double mutants aged between 2 and 9 months, whereas $\sim 20-30$ and $50 \%$ of all axons were unmyelinated in age-matched MAG- and Fyn-deficient mice, respectively. A possible explanation of this finding is that MAG and Fyn act independently to initiate myelination; the severe hypomyelination of the double mutant would simply reflect a phenotypic combination of the respective single mutants. However, Fyn has been demonstrated to act as a signaling molecule downstream of L-MAG (Umemori et al., 1994). Thus, compensatory mechanisms might explain why the double mutant is more severely affected than each of the single mutants. In the absence of Fyn, other signaling pathways might be activated more efficiently by MAG to initiate myelination. The demonstration of an L-MAGassociated kinase activity in Fyn null mutants at early stages of myelination is of potential interest in this context (Umemori et al., 1994). Vice versa, in the absence of MAG, Fyn might be activated more strongly by other oligodendrocyte cell surface molecules (see above). Simultaneous elimination of both MAG and Fyn would disrupt these hypothetical compensatory mechanisms, resulting in a phenotype of the double mutant that is more severe than that of each of the single mutants.

Normal numbers of oligodendrocytes in optic nerves of different genotypes demonstrate that hypomyelination in the mutants does not result from death of oligodendrocytes. Thus, hypomyelination results from either impaired interactions between myelinating oligodendrocyte processes and axons or an impaired morphological maturation of oligodendrocytes. Support for the latter possibility is provided by the recent finding that morphological maturation of oligodendrocytes requires Fyn kinase activity (Osterhout et al., 1999). The authors demonstrated a significant increase in Fyn tyrosine kinase activity during early steps of oligodendrocyte progenitor cell differentiation. Importantly, the increase in Fyn kinase activity preceded morphological maturation of oligodendrocytes characterized by the elaboration of a complex network of processes. Interestingly, pharmacological inhibition of Fyn kinase activity prevented morphological maturation of oligodendrocytes by interfering with the formation of cellular processes. Similar results were obtained when a dominant-negative form of Fyn was introduced into the cells (Osterhout et al., 1999). Given the association of NCAM120 and Fyn in DIGs of oligodendrocytes and myelin (see above), it is interesting that NCAM substrates stimulate formation of myelin-like membranes by differentiating oligodendrocytes in vitro (Gard et al., 1996). Fascicles of unmyelinated axons without interdigitating glial cell processes, evident in Fyn and MAG/Fyn null mutants, suggest that impaired process formation of mutant oligodendrocytes might indeed be the major cause of hypomyelination. It is interesting in this context that we observed a considerable variability in the degree of hypomyelination between different CNS structures of MAG/Fyn double mutants. Our observations demonstrate that tracts containing relatively small-sized ax- ons (i.e., optic nerve and corticospinal tract) are massively hypomyelinated in the double mutant, whereas white matter containing larger axons (i.e., fasciculus gracilis and cuneatus or ventral funiculus) is significantly less affected. A single oligodendrocyte myelinates numerous small-sized axons but only a few or only one large-sized axon. In the latter case, oligodendrocyte cell bodies might be directly associated with the axon, without an interconnecting process (Hildebrand et al., 1993). Along these lines, impaired formation of oligodendrocyte processes can be expected to cause a more pronounced hypomyelination in tracts with smallsized axons than in tracts with large-sized axons. In the future, it will be interesting to visualize the cytoarchitecture of individual oligodendrocytes in the different mutants by performing, for instance, intracellular injections of dyes (Ransom et al., 1991; Butt et al., 1994). Only moderate hypomyelination of some axon tracts of MAG/Fyn double mutants might also explain the relatively mild behavioral deficits of these animals.

The ultrastructural analysis of myelin sheaths of MAG-deficient mice revealed a variety of morphological abnormalities, including an impaired development of the periaxonal oligodendrocyte cytoplasmic collar, presence of noncompacted regions of myelin, formation of redundant myelin, degenerative alterations of myelin sheaths, or the presence of multiply myelinated axons (Li et al., 1994; Montag et al., 1994; Bartsch et al., 1995; Lassmann et al., 1997). Interestingly, noncompacted regions of myelin, redundant myelin, and multiply myelinated axons were also detected in the CNS of mutant mice deficient in the large isoform of MAG (Fujita et al., 1998). These observations raise the possibility that Fyn as a signaling molecule downstream of L-MAG might be involved in the formation of ultrastructurally intact myelin sheaths. However, morphologically abnormal myelin sheaths typically seen in MAG null mutants or L-MAG-deficient mice were not observed in Fyn null mutants. In fact, quantitative analysis revealed no significant difference in the ultrastructure of myelin sheaths between Fyn mutants and wild-type mice. Moreover, myelin sheaths of MAG/ Fyn double mutants showed similar defects with a similar frequency as myelin sheaths of MAG null mutants. All these data demonstrate that Fyn is not involved in the formation of morphologically intact CNS myelin and thus indicate that other signaling molecules downstream of MAG are involved in the morphological maturation of CNS myelin. Formation of morphologically intact myelin in the absence of Fyn is also remarkable in a different context; Fyn has been demonstrated to positively regulate expression of myelin basic protein (MBP), and levels of MBP protein are massively reduced in Fyn null mutants (Umemori et al., 1999).

Because Fyn is expressed by Schwann cells (Bare et al., 1993), the PNS of different mutants was also analyzed. Different from the CNS, ultrastructural analysis of peripheral nerves of adult Fyn null mutants did not reveal evidence for an impaired initiation of myelination. The ultrastructure of myelin sheaths and the longterm maintenance of axon-myelin units was also not affected in the PNS of Fyn mutants until 9 months of age, the oldest animals studied (S. Carenini, D. Montag, M. Schachner, and R. Martini, unpublished data). Thus, MAG-related signaling pathways mediating long-term integrity of axon-myelin units in the PNS or leading to maturation of ultrastructurally intact myelin sheaths in the CNS remain to be elucidated.

\section{REFERENCES}

Arquint M, Roder J, Chia LS, Down J, Wilkinson D, Bayley H, Braun P, Dunn R (1987) Molecular cloning and primary structure of myelinassociated glycoprotein. Proc Natl Acad Sci USA 84:600-604.

Bare DJ, Lauder JM, Wilkie MB, Maness PF (1993) p59fyn in rat brain is localized in developing axonal tracts and subpopulations of adult neurons and glia. Oncogene 8:1429-1436.

Bartsch U (1996) Myelination and axonal regeneration in the central nervous system of mice deficient in the myelin-associated glycoprotein. J Neurocytol 25:303-313.

Bartsch U, Kirchhoff F, Schachner M (1989) Immunohistological localization of the adhesion molecules L1, N-CAM, and MAG in the developing and adult optic nerve of mice. J Comp Neurol 284:451-462.

Bartsch S, Bartsch U, Dörries U, Faissner A, Weller A, Ekblom P, 
Schachner M (1992) Expression of tenascin in the developing and adult cerebellar cortex. J Neurosci 12:736-749.

Bartsch U, Faissner A, Trotter J, Dörries U, Bartsch S, Mohajeri H, Schachner M (1994) Tenascin demarcates the boundary between the myelinated and nonmyelinated part of retinal ganglion cell axons in the developing and adult mouse. J Neurosci 14:4756-4768.

Bartsch U, Montag D, Bartsch S, Schachner M (1995) Multiply myelinated axons in the optic nerve of mice deficient for the myelin-associated glycoprotein. Glia 14:115-122.

Bartsch S, Montag D, Schachner M, Bartsch U (1997) Increased number of unmyelinated axons in optic nerves of adult mice deficient in the myelin-associated glycoprotein (MAG). Brain Res 762:231-234.

Berglund EO, Murai KK, Fredette B, Sekerkova G, Marturano B, Weber L, Mugnaini E, Ranscht B (1999) Ataxia and abnormal cerebellar microorganization in mice with ablated contactin gene expression. Neuron 24:739-750.

Bhat S, Silberberg DH (1986) Oligodendrocyte cell adhesion molecules are related to neural cell adhesion molecule (N-CAM). J Neurosci 6:3348-3354.

Butt AM, Colquhoun K, Tutton M, Berry M (1994) Three-dimensional morphology of astrocytes and oligodendrocytes in the intact mouse optic nerve. J Neurocytol 23:469-485.

Cremer H, Lange R, Christoph A, Plomann M, Vopper G, Roes J, Brown R, Baldwin S, Kraemer P, Scheff S, Barthels D, Rajewsky K, Wille W (1994) Inactivation of the N-CAM gene in mice results in size reduction of the olfactory bulb and deficits in spatial learning. Nature 367:455-459.

Fanarraga ML, Dickinson PJ, Sommer I, Montague P, Kyriakides E, Griffiths IR (1996) Evidence that some oligodendrocyte progenitors in the developing optic pathway express the plp gene. Glia 18:282-292.

Fujita N, Kemper A, Dupree J, Nakayasu H, Bartsch U, Schachner M, Maeda N, Suzuki K, Suzuki K, Popko B (1998) The cytoplasmic domain of the large myelin-associated glycoprotein isoform is needed for proper CNS but not peripheral nervous system myelination. J Neurosci 18:1970-1978.

Gard AL, Maughon RH, Schachner M (1996) In vitro oligodendrogliotrophic properties of cell adhesion molecules in the immunoglobulin superfamily: myelin-associated glycoprotein and N-CAM. J Neurosci Res 46:415-426.

Hildebrand C, Remahl S, Persson H, Bjartmar C (1993) Myelinated nerve fibers in the CNS. Prog Neurobiol 40:319-384.

Koch T, Brugger T, Bach A, Gennarini G, Trotter J (1997) Expression of the immunoglobulin superfamily cell adhesion molecule $\mathrm{F} 3$ by oligodendrocytelineage cells. Glia 19:199-212.

Krämer E-M, Klein C, Koch T, Boytinck M, Trotter J (1999) Compartmentation of Fyn kinase with glycosylphosphatidylinositol-anchored molecules in oligodendrocytes facilitates kinase activation during myelination. J Biol Chem 274:29042-29049.

Laeng P, Molthagen M, Yu EG-X, Bartsch U (1996) Transplantation of oligodendrocyte progenitor cells into the rat retina: extensive myelination of retinal ganglion cell axons. Glia 18:200-210.

Lai C, Brow MA, Nave K-A, Noronha AB, Quarles RH, Bloom FE, Milner
RJ, Sutcliffe JG (1987) Two forms of 1B236/myelin-associated glycoprotein, a cell adhesion molecule for postnatal neural development, are produced by alternative splicing. Proc Natl Acad Sci USA 84:4337-4341.

Lassmann H, Bartsch U, Montag D, Schachner M (1997) Dying-back oligodendrogliopathy: a late sequel of myelin associated glycoprotein deficiency. Glia 19:104-110.

Li C, Tropak MB, Gerial R, Clapoff S, Abramow-Newerly W, Trapp B, Peterson A, Roder J (1994) Myelination in the absence of myelinassociated glycoprotein. Nature 369:747-750.

Montag D, Giese KP, Bartsch U, Martini R, Lang Y, Blüthmann H, Karthigasan J, Kirschner DA, Wintergerst ES, Nave K-A, Zielasek J, Toyka KV, Lipp H-P, Schachner M (1994) Mice deficient for the myelin-associated glycoprotein show subtle abnormalities in myelin. Neuron 13:229-246.

Nave K-A, Lai C, Bloom FE, Milner RJ (1986) Jimpy mutant mouse: a 74-base deletion in the mRNA for myelin proteolipid protein and evidence for a primary defect in RNA splicing. Proc Natl Acad Sci USA 83:9264-9268.

Osterhout DJ, Wolven A, Wolf RM, Resh MD, Chao MV (1999) Morphological differentiation of oligodendrocytes requires activation of Fyn tyrosine kinase. J Cell Biol 145:1209-1218.

Pedraza L, Frey AB, Hempstead BL, Colman DR, Salzer JL (1991) Differential expression of MAG isoforms during development. J Neurosci Res 29:141-148.

Ransom BR, Butt AM, Black JA (1991) Ultrastructural identification of HRP-injected oligodendrocytes in the intact rat optic nerve. Glia 4:37-45.

Salzer JL, Holmes WP, Colman DR (1987) The amino acid sequences of the myelin-associated glycoproteins: homology to the immunoglobulin gene superfamily. J Cell Biol 104:957-965.

Schachner M, Bartsch U (2000) Multiple functions of the myelin-associated glycoprotein MAG (siglec-4a) in formation and maintenance of myelin. Glia 29:154-165.

Stein PL, Lee H-M, Rich S, Soriano P (1992) pp59fyn mutant mice display differential signaling in thymocytes and peripheral T cells. Cell 70:741-750

Timsit S, Martinez S, Allinquant B, Peyron F, Puelles L, Zalc B (1995) Oligodendrocytes originate in a restricted zone of the embryonic ventral neural tube defined by DM-20 mRNA expression. J Neurosci 15:1012-1024.

Tropak MB, Johnson PW, Dunn RJ, Roder JC (1988) Differential splicing of MAG transcripts during CNS and PNS development. Mol Brain Res 4:143-155.

Trotter J, Bitter-Suermann D, Schachner M (1989) Differentiation-regulated loss of the polysialylated embryonic form and expression of the different polypeptides of the neural cell adhesion molecule by cultured oligodendrocytes and myelin. J Neurosci Res 22:369-383.

Umemori H, Sato S, Yagi T, Aizawa S, Yamamoto T (1994) Initial events of myelination involve fyn tyrosine kinase signaling. Nature 367:572-576.

Umemori H, Kadowaki Y, Hirosawa K, Yoshida Y, Hironaka K, Okano H, Yamamoto T (1999) Stimulation of myelin basic protein gene transcription by fyn tyrosine kinase for myelination. J Neurosci 19:1393-1397. 